\title{
Upper-Extremity Function Predicts Adverse Health Outcomes among Older Adults Hospitalized for Ground-Level Falls
}

\author{
Bellal Joseph ${ }^{\mathrm{a}}$ Nima Toosizadeh ${ }^{\mathrm{b}-\mathrm{e}}$ Tahereh Orouji Jokar ${ }^{\mathrm{a}}$ \\ Michelle R. Heusser ${ }^{b, e, f}$ Jane Mohler ${ }^{a, b}, d, f$ Bijan Najafi ${ }^{a, b}, f, g$ \\ ${ }^{a}$ Division of Trauma, Critical Care, Burn and Emergency Surgery and ${ }^{\mathrm{b}}$ Interdisciplinary Consortium on Advanced \\ Motion Performance (iCAMP), Department of Surgery, ${ }^{\mathrm{C}}$ Arizona Center on Aging and ${ }^{\mathrm{d}}$ Division of Geriatrics, General \\ Internal Medicine and Palliative Medicine, Department of Medicine, and 'Department of Biomedical Engineering, \\ University of Arizona, Tucson, AZ, ${ }^{f}$ Department of Bioengineering, University of Pittsburgh, Pittsburgh, PA, and \\ 9 Interdisciplinary Consortium on Advanced Motion Performance (iCAMP), Michael E. DeBakey Department of \\ Surgery, Baylor College of Medicine, Houston, TX, USA
}

\section{Keywords}

Frailty - Discharge disposition · Readmission · Inpatient care - Trauma · Fall incident - Wearable technology . Functional test · Bedbound patients · Inpatient triage

\begin{abstract}
Background: Despite National Surgical Quality Improvement guidelines to integrate frailty into surgical elder assessments, a quick, accurate, and simple frailty assessment tool suitable for busy clinical settings is still not available. Recently, we have demonstrated that a simple upper-extremity function (UEF) test based on wearable sensors could identify frailty with high agreement with conventional assessments by testing 20-s repetitive elbow flexion and extension. $\mathbf{O b}$ jective: We examined whether UEF parameters are sensitive for predicting adverse health outcomes in bedbound older adults admitted to hospital due to ground-level fall injuries. Study Design: Frailty was assessed in 101 eligible older adults (age: $79 \pm 9$ years) admitted to a trauma setting using the UEF test at the time of admission. All participants were followed up for 2 months using phone calls and chart reviews. The measured health outcomes included (1) dis-
\end{abstract}

\section{KARGER}

(C) 2016 S. Karger AG, Basel

E-Mail karger@karger.com

www.karger.com/ger charge disposition (favorable: discharge home or rehabilitation; unfavorable: discharge to skilled nursing facility or death), (2) hospital length of stay, (3) 30-day readmission, (4) 60-day readmission, and (5) 30-day prospective falls. Multivariate analyses were used to identify independent predictors of adverse health outcomes based on participants' demographic parameters (i.e., age, gender, and body mass index [BMI]) and UEF index. Results: Based on the UEF frailty status, 53 (52\%) of the participants were frail and 48 (48\%) were non-frail. Among all adverse health outcomes, age was only a significant predictor of 30-day prospective falls ( $p=$ 0.023). On the other hand, the UEF index was a significant predictor of all measured outcomes except hospital length of stay $(p<0.010)$. Among the UEF parameters, those indicating slowness, weakness, and exhaustion had the highest effect sizes to predict an unfavorable discharge disposition ( $p<0.010$; effect size $=0.65-0.92$ ). Conclusion: The results of this study suggest that a 20 -s UEF test is practical in the

Bijan Najafi, PhD

Interdisciplinary Consortium on Advanced Motion Performance (iCAMP)

Michael E. DeBakey Department of Surgery, Baylor College of Medicine

One Baylor Plaza, MS: BCM390, Houston, TX 77030 (USA)

E-Mail bijan.najafi@bcm.edu

Bellal Joseph, MD

Division of Trauma, Critical Care, Burn and Emergency Surgery

Department of Surgery, University of Arizona

Tucson, AZ 85724 (USA)

E-Mail bjoseph@surgery.arizona.edu 
trauma setting and could be used as a quick measure for predicting adverse events and outcomes among bedbound patients after discharge. Assessing frailty using UEF may assist in objective triage, treatment, and post-discharge decisionmaking with regard to geriatric trauma patients.

C 2016 S. Karger AG, Basel

\section{Introduction}

Falls and fall-related injuries are significant health and safety problems for older adults. Nearly $30 \%$ of community dwellers over the age of 65 years experience 1 or more falls every year, leading to a significant risk for hospitalization, institutionalization, and even death [1]. Of older adults who are hospitalized for falls, only half survive, and the other half succumb to the trauma a year later [2]. Although age is strongly associated with adverse health outcomes due to traumatic falls, the response of an older adult to traumatic events, hospitalization, treatment, and rehabilitation is highly variable due to the heterogeneity of aging [3]. To account for this variation, risk stratification using a measure of physiological function is required. Recently, measures of frailty have been used to predict low physiological functional reserve and high vulnerability to poor outcomes $[4,5]$.

We previously developed an objective method for assessing frailty based on upper-extremity function (UEF) and validated this test among trauma patients with convergence to the Trauma-Specific Frailty Index (TSFI) [6, 7]. The UEF test incorporates several frailty features. With UEF, the strength of upper-extremity muscles in performing a rapid task can be measured, thereby resembling the grip strength test. Also, the theorized biological mechanism is that sarcopenia/dynapenia, as a manifestation of physical frailty, would influence the entire muscular structure of the human body, including both the lower and the upper extremities $[4,8]$. Therefore, speed of upper-extremity movement was measured as a surrogate for gait speed. The UEF assessment method integrates low-cost sensors, the physical task (elbow flexion) is easily performed in under $1 \mathrm{~min}$, and the postprocessing is performed in less than 2 min.

In a recent validation study among 101 trauma patients ( $\geq 65$ years), the UEF index was developed to include speed of elbow flexion (slowness), elbow power (weakness), and speed reduction (exhaustion) in a repetitive 20-s elbow flexion test [7]. The purpose of the current prospective study was to assess the validity of the previously developed UEF index (based on the TSFI) in predicting in-hospital and post-discharge health outcomes in 101 older adult patients (aged $\geq 65$ years) admitted for ground-level falls to a southwestern academic integrated health care system level I trauma center. We hypothesized that the UEF index would be significantly associated with discharge disposition, readmission, and prospective falls.

\section{Subjects and Methods}

\section{Participants}

Older adults with ground-level fall injuries were recruited (January 2014 to August 2015) from the Division of Trauma, Critical Care, Burn and Emergency Surgery service at the University of Arizona. A fall was defined as an incident in which a participant unintentionally came to rest on a lower surface $[9,10]$. The inclusion criteria were (1) being aged 65 years and older, (2) possessing the ability to understand the study instructions for performing repetitive elbow flexion, and (3) having had at least 1 groundlevel fall causing an injury within the prior 2 weeks that led to hospital admission. Participants were excluded if they had significant upper-extremity disorders in both arms (e.g., bilateral fractures or rheumatoid arthritis with elbow or shoulder involvement), or if they were in a physical condition (e.g., such as severe head injury and unconsciousness) that precluded them from performing the UEF test. Participants were not screened for cognition, since cognitive impairment is a common comorbidity among older adults and is associated with falls and adverse health outcomes [11-13].

The study was approved by the University of Arizona Institutional Review Board. Written informed consent was obtained by trained research coordinators according to the principles expressed in the Declaration of Helsinki [14] from all participants, or from their authorized health care power of attorney if a participant was clinically assessed as lacking capacity for informed consent.

\section{Health Outcome Assessment}

All participants were followed up for 2 months using monthly phone calls and chart reviews. The measured health outcomes included (1) discharge disposition (favorable: discharge home or rehabilitation; unfavorable: discharge to skilled nursing facility or death), (2) hospital length of stay, (3) 30-day readmission, (4) 60 -day readmission, and (5) 30-day prospective falls. Readmission and fall events were assessed from the day of discharge. Of note, all readmissions to the same center or other centers due to trauma and falls were recorded. Further, 30- and 60-day readmission and 30-day prospective fall events were confirmed by a relative or an authorized caregiver in case participants were not able to recall them. Additionally, comorbid conditions were recorded, including hypertension, stroke, blood dyscrasia, hypercholesterolemia, cirrhosis, chronic obstructive pulmonary disease, history of cancer, use of immunosuppressive medications, autoimmune disorders, coronary artery disease, dementia, and chronic renal failure. The injury severity score (ISS) and the Abbreviated Injury Scale (AIS) score were also recorded to assess the severity of injury. 
Table 1. Definitions of UEF parameters

\begin{tabular}{ll}
\hline Parameter & Definition \\
\hline Speed & Mean value of the elbow angular velocity range (maximum minus minimum speed) \\
\hline Flexibility & Mean value of the elbow flexion range \\
\hline Power & $\begin{array}{l}\text { Mean value of the product of the angular acceleration range and the range of angular } \\
\text { velocity }\end{array}$ \\
\hline Rise time & Mean value of the time required to reach maximum angular velocity \\
\hline Moment & $\begin{array}{l}\text { Mean value of the maximum moments on the elbow within each flexion/extension } \\
\text { estimated from the moment of inertia of the forearm and hand, and elbow motion }\end{array}$ \\
\hline Speed variability & $\begin{array}{l}\text { Coefficient of variation (standard deviation divided by the mean) of the angular velocity } \\
\text { range }\end{array}$ \\
\hline Speed reduction & $\begin{array}{l}\text { Difference in angular velocity range between the last and the first } 5 \mathrm{~s} \text { of elbow flexion as a } \\
\text { percentage of the initial angular velocity range }\end{array}$ \\
\hline Flexion number & \begin{tabular}{l} 
Number of flexions/extensions during $20 \mathrm{~s}$ \\
\hline
\end{tabular}
\end{tabular}

Of the UEF parameters, speed, power, and speed reduction were used to develop the UEF index. UEF, upperextremity function.

\section{UEF Assessment and Index}

Per our previously validated method [15], wearable sensor technology was used to measure forearm and upper-arm motion. A triaxial wearable gyroscope sensor (dynamic range: $\pm 2,000 \%$ s; sample frequency: $100 \mathrm{~Hz}$; BioSensics LLC, Boston, MA, USA) was attached to the upper arm near the biceps, and one to the wrist, using a band attached with Velcro, to estimate 3-dimensional angular velocity of the upper-arm and forearm segments, and ultimately elbow angular velocity. Each participant performed an approximately 20 -s trial of elbow flexion, within which they repetitively flexed and extended their dominant elbow to full flexion and extension as quickly as possible in supine (bedbound) position while wearing the UEF system. Participants performed the elbow flexion task with their nondominant side in the case of injury or attachments of vital sign-monitoring equipment. Before the actual test, participants performed a short practice trial to become familiar with the protocol. The protocol was explained to the participants, and they were encouraged only once, before elbow flexion, to do the task as fast as possible (they were encouraged no further during the task). All UEF assessments were performed by trained researchers (N.T., M.R.H., and T.O.J.) during the participants' hospital stay.

Several outcome measures representing kinematics and kinetics of elbow flexion were derived using angular velocity and anthropometric data (i.e., the participants' stature and body mass). The outcome measures included (1) speed, (2) flexibility, (3) power, (4) rise time, (5) moment, (6) speed variability, (7) speed reduction, and (8) flexion number (see Table 1 for definitions) [15]. The UEF index represents the continuous frailty index and categorical frailty status (i.e., non-frail and frail) based on the UEF parameters and has been validated using a modified Rockwood questionnaire, the TSFI [6]. The results from the validation study showed a high agreement $(r=0.72, p<0.0001)$ between TSFI score and UEF model; sensitivity and specificity for predicting the frailty status were 78 and $82 \%$, respectively, compared to the TSFI as the gold standard. Readers are referred to previous work $[7,15]$ for more details regarding the validation of

Frailty Measurement to Predict Adverse Health Outcomes in Bedbound Elderly
UEF using a motion capture system, and for a detailed description of parameter calculations and UEF index development. Briefly, the UEF index is a 0 -to- 1 score $(0=$ minimum frailty symptoms; 1 = maximum frailty symptoms), and those with a score of $\leq 0.27$ are considered non-frail (those with a score of $>0.27$ are frail). Of note, only speed, power, and speed reduction (reduction in angular velocity within $20 \mathrm{~s}$ ) were used to develop the UEF index, as they showed the best associations with the TSFI. These parameters, respectively, represent "slowness," "weakness," and "exhaustion" frailty markers.

\section{Statistical Analysis}

Between-group differences in demographic information and adverse health outcomes for the frailty groups (i.e., non-frail: UEF index $\leq 0.27$; frail: UEF index $>0.27$ ) were determined using analyses of variance (ANOVAs) and Cohen's effect sizes were calculated [16]; statistical analyses were adjusted for age, gender, and BMI for comparisons of adverse health outcomes between the frailty groups. Further, separate models of logistic regression (for nominal health outcomes) and ANOVA regression (for continuous health outcomes) were used to determine the association between the UEF index (as one of the independent variables) with each of the health outcomes (as a dependent variable); all regressions were adjusted for age, gender, and BMI (a total of 5 models).

Statistical analyses were adjusted for age, gender, and BMI to account for possible influences of these parameters on health outcomes. For those adverse health outcomes that showed a significant association with the UEF index, we performed further statistical analyses to determine the association between individual UEF parameters with each of the adverse health outcomes. For this purpose, the UEF parameters were compared between 2 groups (with positive or negative health outcomes) using separate ANOVAs with age, gender, and BMI as covariates. To account for the effect of discharge disposition (favorable vs. unfavorable) on the results, additional logistic regression models were developed to associate the UEF index with 30- and 60-day readmission and 30-day prospective falls, adjusting for age and discharge disposition. All anal- 
Table 2. Demographic data and health outcome measures for the frailty groups as defined by the UEF test

\begin{tabular}{|c|c|c|c|c|c|c|}
\hline Variable & Non-frail group & Frail group & $p$ value $^{\mathrm{a}}$ & Lower CI & Upper CI & Effect size \\
\hline Number, $n$ (\% of total) & $48(48)$ & $53(52)$ & - & - & - & - \\
\hline Mean UEF index (SD) & $0.18(0.05)$ & $0.39(0.10)$ & - & - & - & - \\
\hline Male, $n$ ( $\%$ of the group) & $26(54)$ & $21(40)$ & 0.143 & -0.10 & 0.69 & - \\
\hline Mean age (SD), years & $76(9)$ & $81(9)$ & $0.004^{*}$ & 0.88 & 4.39 & 0.56 \\
\hline Mean stature (SD), $\mathrm{cm}$ & $168.32(9.41)$ & $166.48(10.36)$ & 0.360 & -2.91 & 1.07 & 0.19 \\
\hline Mean body mass (SD), $\mathrm{kg}$ & $72.72(15.14)$ & $68.84(15.98)$ & 0.221 & -5.06 & 1.18 & 0.25 \\
\hline Mean BMI (SD) & $25.55(4.40)$ & $24.74(4.84)$ & 0.388 & -1.33 & 0.52 & 0.18 \\
\hline Unfavorable discharge disposition, & & & & & & \\
\hline $\begin{array}{l}n \text { (\% of the group) } \\
\text { Mean hospital length of stay (SD) davs }\end{array}$ & $\begin{array}{c}8(17) \\
444(298)\end{array}$ & $\begin{array}{l}24(45) \\
585(531)\end{array}$ & $0.003^{*}$ & $\begin{array}{r}0.25 \\
-0.33\end{array}$ & $\begin{array}{l}1.32 \\
1.54\end{array}$ & - \\
\hline 30 -day readmission, $n$ (\% of the group) & $10(21)$ & $19(36)$ & 0.136 & -0.85 & 0.11 & - \\
\hline 60 -day readmission, $n$ (\% of the group) & $15(31)$ & $28(53)$ & 0.097 & -0.80 & 0.07 & - \\
\hline 30 -day prospective falls, $n$ (\% of the group) & $5(10)$ & $16(30)$ & $0.049 *$ & -1.14 & 0.04 & - \\
\hline
\end{tabular}

UEF, upper-extremity function. * Significant between-group difference.

${ }^{a} p$ values for between-group differences in adverse health outcome measures are adjusted for age, gender, and BMI.

Table 3. Comorbidity measures for the frailty groups as defined by the UEF test

\begin{tabular}{lclc}
\hline Comorbidities & $\begin{array}{l}\text { Non-frail } \\
\text { group, \% } \\
(n=48)\end{array}$ & $\begin{array}{l}\text { Frail } \\
\text { group, \% } \\
(n=53)\end{array}$ & $p$ value \\
\hline Hypertension & 60 & 92 & $<0.001^{*}$ \\
Hyperlipidemia & 43 & 56 & 0.056 \\
Coronary artery disease & 25 & 46 & $0.001^{*}$ \\
Stroke & 4 & 13 & $0.018^{*}$ \\
Dementia & 23 & 45 & $<0.001^{*}$ \\
Cirrhosis & $<1$ & 1 & 0.436 \\
Chronic renal failure & 8 & 7 & 0.546 \\
Chronic obstructive pulmonary & & & \\
\multicolumn{1}{c}{ disease } & 3 & 18 & $<0.001^{*}$ \\
\hline
\end{tabular}

UEF, upper-extremity function. * Significant between-group difference.

yses were performed using JMP ${ }^{\mathrm{TM}}$ (version 11; SAS Institute Inc., Cary, NC, USA), with a significance level of $p<0.05$. Summary results are presented as means with standard deviation (SD) and standard error (SE).

\section{Results}

\section{Participants}

A total of 101 older adults were recruited for the current study. Based on the UEF frailty status, 53 (52\%) of
Table 4. Injury details for the frailty groups as defined by the UEF test

\begin{tabular}{llll}
\hline Variable & $\begin{array}{l}\text { Non-frail } \\
\text { group } \\
(n=48)\end{array}$ & $\begin{array}{l}\text { Frail } \\
\text { group } \\
(n=53)\end{array}$ & $p$ value \\
\hline Mechanism, \% & & & \\
$\quad$ Blunt injury & 96 & 96 & 0.608 \\
$\quad$ Fall & 53 & 68 & $0.020^{*}$ \\
$\quad$ Motor vehicle collision & 40 & 26 & $0.022^{*}$ \\
ISS & $10(10-14)$ & $10(9-14)$ & 0.220 \\
Head-AIS score & $2(2-3)$ & $2(2-3)$ & 0.082 \\
Thorax-AIS score & $3(2-3)$ & $3(2-3)$ & 0.550 \\
Abdomen-AIS score & $2(2-2)$ & $2(1-2)$ & 0.124 \\
Extremity-AIS score & $2(2-3)$ & $2(1-2)$ & 0.123 \\
\hline
\end{tabular}

Values for the ISS and AIS are medians (interquartile ranges) of the injury scores. ISS, injury severity score; AIS, Abbreviated Injury Scale. * Significant between-group difference.

the participants were frail and 48 (48\%) were non-frail. The mean (SD) age and BMI of the frail participants were 81 (9) years and 24.74 (4.84); the corresponding values were 76 (9) years and 25.55 (4.40) for the non-frail participants. Of the demographic parameters, only age was significantly different between the UEF-defined non-frail and frail groups $(p=0.004)$. Other sociodemographic data are reported in Table 2. Overall, frail participants were more likely to have pre-existing comorbid conditions than non-frail patients. Table 3 provides a comparison of several pre-existing comorbidities between the
Joseph/Toosizadeh/Orouji Jokar/Heusser/ Mohler/Najafi 
Table 5. Association between the UEF index and prospective health outcomes

\begin{tabular}{llccccc}
\hline Independent variable & Dependent variable & $\begin{array}{l}\text { Parameter } \\
\text { estimate }\end{array}$ & $\chi^{2}$ or $t$ ratio & $p$ value & $\begin{array}{l}\text { Lower } \\
\text { CI }\end{array}$ & $\begin{array}{l}\text { Upper } \\
\text { CI }\end{array}$ \\
\hline UEF index (0-1) & Unfavorable discharge & 9.23 & 14.58 & $<0.001^{*}$ & 4.84 & 14.40 \\
Age (years) & disposition & 0.01 & 0.07 & 0.794 & -0.05 & 0.07 \\
Gender (female) & & 0.15 & 0.31 & 0.578 & -0.38 & 0.68 \\
BMI & & 0.04 & 0.45 & 0.498 & -0.07 & 0.15 \\
\hline UEF index (0-1) & Hospital length of stay & 4.16 & 1.14 & 0.256 & -3.06 & 11.37 \\
Age (years) & & 0.02 & 0.41 & 0.681 & -0.08 & 0.12 \\
Gender (female) & & -0.27 & -0.56 & 0.576 & -1.21 & 0.68 \\
BMI & & -0.14 & -1.41 & 0.162 & -0.34 & 0.06 \\
\hline UEF index (0-1) & 30-day readmission & 4.88 & 6.65 & $0.008^{*}$ & 1.28 & 8.78 \\
Age (years) & & 0.01 & 0.06 & 0.808 & -0.05 & 0.06 \\
Gender (female) & & -0.32 & 1.71 & 0.186 & -0.82 & 0.15 \\
BMI & & -0.01 & 0.01 & 0.939 & -0.11 & 0.10 \\
\hline UEF index (0-1) & 60-day readmission & 4.44 & 6.11 & $0.010^{*}$ & 1.04 & 8.14 \\
Age (years) & & 0.02 & 0.92 & 0.345 & -0.02 & 0.07 \\
Gender (female) & & -0.21 & 0.84 & 0.356 & -0.67 & 0.23 \\
BMI & & 0.01 & 0.01 & 0.935 & -0.09 & 0.10 \\
\hline UEF index (0-1) & 30-day prospective falls & 5.55 & 6.23 & $0.010^{*}$ & 1.31 & 10.14 \\
Age (years) & & 0.07 & 4.47 & $0.023^{*}$ & 0.01 & 0.14 \\
Gender (female) & & -0.56 & 3.42 & 0.056 & -1.18 & 0.01 \\
BMI & & -0.11 & 2.39 & 0.103 & -0.25 & 0.02 \\
\hline
\end{tabular}

Independent variables for each regression analysis included the UEF index, age, gender, and BMI. UEF, upperextremity function. * Significant independent association. ${ }^{\text {a }}$ Parameter estimates for nominal health outcomes are presented for log odds of adverse event presence/absence.

groups. Blunt injury was the predominant mechanism of injury in our population. Frail participants were more likely to present with falls than non-frail patients $(p=$ 0.02 ). Table 4 elaborates the comparison of injury patterns and severity.

\section{Association between UEF and Adverse Health \\ Outcomes}

As indicated in Table 2, the percentage of participants who had adverse prospective health outcomes following their hospital stay was greater in the frail than in the non-frail group as defined by UEF; differences were significant for the rates of an unfavorable discharge disposition and prospective falls within the 30-day follow-up period $(p<0.049)$. Further, when adjusted for sociodemographic data, the UEF index was a significant predictor of all adverse health outcomes $(p<0.010)$ except of hospital length of stay (Table 5); specifically, the UEF index was a strong predictor of an unfavorable discharge

Frailty Measurement to Predict Adverse Health Outcomes in Bedbound Elderly disposition $(p<0.001)$ and readmission $(p<0.010)$. When the logistic models were adjusted for discharge disposition (in addition to age), the UEF index remained a significant predictor of 30- and 60-day readmission and of 30-day prospective falls $(p<0.03)$. Of importance, chronological age itself was significantly associated with a single adverse outcome, i.e., prospective falls $(p=0.02$; Table 5$)$.

The ANOVA analyses revealed that all 3 UEF index parameters (i.e., speed, power, and speed reduction) were significantly different between individuals who had an unfavorable discharge disposition and those who had not $(p<0.010$; effect size $=0.65-0.92$; Fig. 1$)$. The ANOVA results for the other adverse health outcome measures, including 30- and 60-day readmission and 30-day prospective falls, showed that only the speed reduction parameter was consistently worse among participants who had adverse health outcomes following hospitalization $(p<0.03$; effect size $=0.42-0.65)$. 


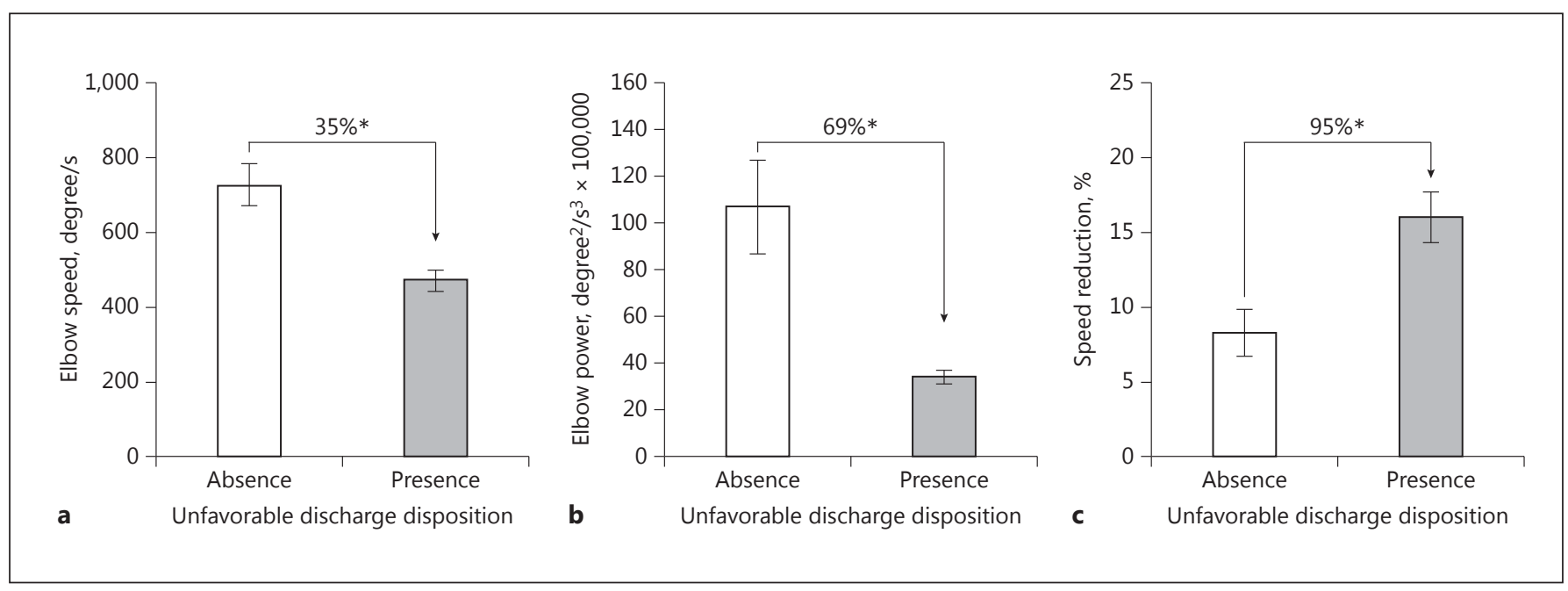

Fig. 1. Differences in the UEF parameters of slowness (a), weakness (b), and exhaustion (c) between participants who had a favorable and those who had an unfavorable discharge disposition following their hospital stay. * Significant difference.

\section{Discussion}

\section{UEF and Frailty}

Although several researchers have focused on identifying frailty, assessing frailty is still controversial [17]. Two common methods for defining frailty are (1) the Fried categorical index, based on a specific phenotype of 5 criteria (weight loss, exhaustion, low physical activity, weak grip strength, and slow walking speed), and (2) the Rockwood frailty index, based on a count of 70 accumulated deficits, including the presence and/or severity of diseases, ability in activities of daily living, and physical signs from clinical and neurological examinations $[4,5]$. Among more than 2,000 older adults ( $\geq 70$ years), previous work showed that the 2 measures of Fried and Rockwood were moderately correlated $(r=0.65)$ [5].

The UEF test was initially developed and validated using the "gold-standard" Fried index among communitydwelling older adults. We subsequently validated the test based on the TSFI among trauma patients, and we developed a trauma-specific index. Since approximately $50 \%$ of the trauma patients were not able to walk at the time of measurement, the Fried test was not performed in this trauma setting [7]. Furthermore, the UEF test was validated in a separate study among trauma patients (in addition to community-dwelling older adults) to account for possible differences in function due to injury severity and medications. Overall, a worse UEF performance was observed among hospitalized compared to nonhospital- ized community-dwelling participants [15] when adjusted for age, gender, and BMI. For instance, speed was around $30 \%$ reduced and the speed reduction was approximately $60 \%$ larger among trauma patients $(p<0.02)$.

\section{UEF and Discharge Disposition}

As hypothesized, within the current sample of hospitalized older adults who had a ground-level fall, prospective post-discharge adverse health outcomes were significantly associated with frailty as measured using the UEF index. Among all prospective adverse health outcomes, the strongest association was observed between UEF and unfavorable discharge disposition. Previous studies on trauma patients revealed that the Rockwood 50-item frailty index (which includes comorbidities, daily activities, health attitude, function, and nutrition factors) is a strong predictor of unfavorable discharge disposition, independent of age [18]. To date, to the best of our knowledge, no other study has utilized admission frailty measures for predicting discharge disposition among groundlevel injury or any type of trauma patients. In other clinical research, AIS, ISS, and the Glasgow Coma Scale (GCS) have been commonly used as predictors of posthospitalization outcomes among trauma patients, with weak correlations $(r<0.34)$ between these injury measures and prospective adverse health outcomes $[19,20]$. Of note, all previously implemented methods for assessing prospective adverse health outcomes in trauma patients, including frailty and injury severity assessments, 
involve subjective questionnaires. To the best of our knowledge, the current study is the first proposing a method for objective physical frailty assessment of geriatric trauma patients.

Among older hospitalized adults with nontraumatic injuries, several researchers have reported a significant association between frailty and discharge disposition after surgery [21-23]. Commonly implemented assessments in these studies include measures of activity of daily living (e.g., Katz score), comorbidity (e.g., Charlson comorbidity index), function (e.g., gait speed and Timed Up and Go test), nutrition (e.g., albumin level), cognition (e.g., Mini-Cog test), and geriatric syndromes (e.g., falls), as well as social factors. Of all these factors, functional measures of frailty have been shown to be more closely associated with the need for post-discharge institutionalization [22, 23]. Performing common frailty function measures such as the gait and Timed Up and Go tests is, however, impossible for most bedbound patients with traumatic injuries, and subjective questionnaires are often time-consuming. Accordingly, Goldstein et al. [24], in a review of frailty measures in emergency medicine settings, highlighted the fact that although the utility of measuring frailty is clear, a practical measure of frailty is lacking. The current study focused on validating an objective, rapid method for prospective adverse health outcome evaluation which is easily performed in the acute care setting.

\section{UEF and Readmission}

The readmission rate is often considered a measure of quality of care. Due to the recent congressional enactment of a Medicare Hospital Readmissions Reduction Program, American hospitals are now penalized for 30day readmissions [25]. Our findings show that while age was not significantly associated with readmission, frailty may, to some extent, predict 30-day and 60-day readmission among older trauma patients. Similar to discharge disposition assessments, there are a limited number of studies regarding readmission prediction among trauma patients. In a recent study, Housley et al. [26] used preinjury medications and comorbidities to assess frailty in trauma patients. Within a large sample of 879 trauma patients, they demonstrated that frailty is a significantly stronger predictor of readmission than age. Several studies have recently been published that show the association between frailty and readmission rates among non-trauma older adults, including heart and elective surgery patients.

Among approaches implemented for assessing frailty, the Fried [27-30] and Rockwood [31,32] indices are the

Frailty Measurement to Predict Adverse Health Outcomes in Bedbound Elderly most common tools. Overall, the Fried index, which involves functional measures in addition to subjective questionnaires, provides better predictions of readmission than the Rockwood score. Within the current study, a significant difference in UEF index was also observed between those who were readmitted to hospital and those who were not $(p<0.010$; effect size $=0.57$ and 0.64 for 30 - and 60-day readmission, respectively). Overall, the current findings and the previous literature suggest that frailty assessment may provide a promising tool for identifying older adults with a higher chance of readmission among those in acute and outpatient care.

\section{UEF and Falls}

The UEF index was associated with both prospective falls (current results) and retrospective falls within the prior year [7]. Although several previous studies have associated fall risk with lack of strength and function in lower-extremity muscles [33-37], the current findings suggest that UEF may also be related to higher rates of falls. This observation suggests that this lack of strength or function seen in older adults may be systematic and related to the dynapenia and sarcopenia seen with aging, influencing both lower- and upper-extremity performance. This hypothesis is in agreement with previous studies that revealed associations of upper-body and lower-extremity muscle strength with fall risk $[34,35]$.

Predicting those who are at higher risk of falling would have important financial and health care benefits. In previous research, poor balance and fall risk were related to lack of physiological reserve and frailty among older adults [4, 37-39]. However, measuring frailty in those who are at higher risk of falling, especially in older adults who are admitted for fall injuries, still requires further research. The use of new, objective technology may play a substantial role in enhancement of frailty assessment. Although our current findings require further confirmation in larger samples, they show promise for a practical implementation of wearable sensor technology and UEF for fall assessment in an outpatient setting.

\section{Limitations}

First, as with measurement limitations in gait-based frailty measures, upper-extremity disability or injury may limit measurements; we found this to be the case for some individuals, who were excluded from the study because they were unable to perform the UEF task. That said, it is likely that this limitation would also apply to measuring grip strength, which would likewise limit measurements within the Fried index. Furthermore, due to upper-ex-

Gerontology 2017;63:299-307

DOI: $10.1159 / 000453593$ 
tremity attachment of vital sign-monitoring equipment within the hospital setting, 32 (32\%) of the participants were not able to perform the UEF test on their dominant arm; thus, the assessment was performed using the nondominant arm. However, this is likely to be less important, as our previous findings have demonstrated similar prediction qualities for assessing frailty using either the dominant or the nondominant arm [15].

Second, several of the screened individuals (35\%) that were admitted to the hospital were not able to perform the UEF test, due to severe head injuries or unconsciousness. Although several fall patients were not able to perform the UEF test, this limitation also exists for other common frailty measures such as the Fried (gait-limited) or Rockwood (memory-limited) indices.

Third, all consecutively admitted trauma patients with ground-level falls were screened for the study. As the purpose of the current study was to predict adverse health outcomes in general geriatric trauma patients, the participants were not stratified based on the type of treatment and medication. Also, no information regarding physical therapy or occupation therapy evaluations and treatments during and after hospitalization was available. However, only 1 trauma surgeon (B.J., who was blinded to the frailty and health outcome results) was assigned to all recruited participants.

Fourth, due to time limitations for trauma patient visits, no extensive subjective questionnaires including depression, comorbidity, and cognition were collected in the current study. The purpose of the study was, however, to predict health outcomes using a general UEF test without considering the source of the functional decline (either comorbidity or cognition). Of note, in our previous work we had observed that cognitive impairment caused a reduction in UEF performance [40].

Lastly, our frailty measure was not able to predict hospital length of stay, probably due to other confounding variables that may influence hospitalization, such as diagnosis-related group reimbursement, surgical processes, and rehabilitation requirements. Also 60-day prospective falls were not considered as an outcome, since some data were missing.

\section{Summary of the Findings and Clinical Implications}

Health outcomes following traumatic fall events vary widely due to the heterogeneity of health conditions among older adults. Risk adjustment is crucial in order to determine the appropriate treatment, support, and discharge of geriatric trauma patients - identifying those who will benefit from intensive and expensive treatments versus those who will best respond to palliation - and to better manage appropriate discharge according to disposition. Within the current study, we developed an objective method for assessing frailty based on speed, strength, and exhaustion levels of UEF. Using this approach, an unfavorable discharge disposition to a skilled nursing facility or death, readmission, and prospective falls was identified among older adults with ground-level fall events. Importantly, chronological age was not a significant predictor of unfavorable discharge disposition and readmission. Among the functional parameters used for developing the UEF index, the level of upper-extremity exhaustion was the only parameter that was consistently different between all adverse health outcome measures following hospitalization.

In conclusion, the current results suggest that a simple UEF measure that can be performed at the bedside would allow clinicians to understand the level of frailty, to help identify older adults with a higher risk of discharge complications and readmission.

\section{Acknowledgements}

We thank Bardiya Zangbar for data collection and clinical coordination. Partial support was provided by the National Institutes of Health/National Institute on Aging (award No. 1R44AG050338-0), the National Institute of Biomedical Imaging and Bioengineering (award No. 1R25EB012973), and the Flinn Foundation (award No. 1907). The content is solely the responsibility of the authors and does not necessarily represent the official views of the sponsors.

\section{Authors' Contributions}

B.J. was responsible for the concept and design, clinical supervision, interpretation of data, and obtaining funding; M.R.H. for patient recruitment, study coordination, acquisition of data, and data analysis; N.T. for the concept and design, study coordination, analysis and interpretation of data, preparation of the manuscript, and statistical analysis; T.O.J. for study coordination and acquisition of data; J.M. for the concept and design and interpretation of data; and B.N. for the concept and design, study supervision, interpretation of data, and obtaining funding. All authors contributed by critically revising the manuscript and gave final approval of the version to be published.

\section{Disclosure Statement}

The Upper Extremity Frailty meter is protected by a patent pending (US Patent 20,150,332,004). The patent is owned by the University of Arizona, and N.T., J.M., and B.N. are listed as coinventors on this patent pending. No other conflict of interest is reported by other authors.
306

Gerontology 2017;63:299-307

DOI: $10.1159 / 000453593$
Joseph/Toosizadeh/Orouji Jokar/Heusser/ Mohler/Najafi 


\section{References}

1 Liu-Ambrose T, Davis JC, Hsu CL, Gomez C, Vertes K, Marra C, Brasher PM, Dao E, Khan KM, Cook W, et al: Action seniors! - Secondary falls prevention in community-dwelling senior fallers: study protocol for a randomized controlled trial. Trials 2015;16:144.

2 Rubenstein LZ: Falls in older people: epidemiology, risk factors and strategies for prevention. Age Ageing 2006;35(suppl 2):ii37ii41.

3 Rowe JW, Kahn RL: Human aging: usual and successful. Science 1987;237:143-149.

4 Fried LP, Tangen CM, Walston J, Newman AB, Hirsch C, Gottdiener J, Seeman T, Tracy R, Kop WJ, Burke G, et al: Frailty in older adults: evidence for a phenotype. J Gerontol A Biol Sci Med Sci 2001;56:M146-M156.

5 Rockwood K, Andrew M, Mitnitski A: A comparison of two approaches to measuring frailty in elderly people. J Gerontol A Biol Sci Med Sci 2007;62:738-743.

6 Joseph B, Pandit V, Zangbar B, Kulvatunyou N, Tang A, O'Keeffe T, Green DJ, Vercruysse G, Fain MJ, Friese RS, Rhee P: Validating trauma-specific frailty index for geriatric trauma patients: a prospective analysis. J Am Coll Surg 2014;219:10-17.e1.

7 Toosizadeh N, Joseph B, Heusser MR, Orouji Jokar T, Mohler J, Phelan HA, Najafi B: Assessing upper-extremity motion: an innovative, objective method to identify frailty in older bed-bound trauma patients. J Am Coll Surg 2016;223:240-248.

8 Manini TM, Clark BC: Dynapenia and aging: an update. J Gerontol A Biol Sci Med Sci 2012; 67:28-40.

9 Mirelman A, Herman T, Brozgol M, Dorfman M, Sprecher E, Schweiger A, Giladi N, Hausdorff JM: Executive function and falls in older adults: new findings from a five-year prospective study link fall risk to cognition. PLoS One 2012;7:e40297.

10 Tinetti ME: Prevention of falls and fall injuries in elderly persons: a research agenda. Prev Med 1994;23:756-762.

11 Sampson EL, Blanchard MR, Jones L, Tookman A, King M: Dementia in the acute hospital: prospective cohort study of prevalence and mortality. Br J Psychiatry 2009;195:6166.

12 Dodson JA, Truong TT, Towle VR, Kerins G, Chaudhry SI: Cognitive impairment in older adults with heart failure: prevalence, documentation, and impact on outcomes. Am J Med 2013;126:120-126.

13 Muir SW, Gopaul K, Montero Odasso MM: The role of cognitive impairment in fall risk among older adults: a systematic review and meta-analysis. Age Ageing 2012;41:299-308.

14 World Medical Association: World Medical Association Declaration of Helsinki: ethical principles for medical research involving human subjects. JAMA 2013;310:2191-2194.
15 Toosizadeh N, Mohler J, Najafi B: Assessing upper extremity motion: an innovative method to identify frailty. J Am Geriatr Soc 2015; 63:1181-1186.

16 Cohen J: Statistical Power Analysis for the Behavioral Sciences, rev ed. New York, Academic Press, 1977.

17 Fisher AL: Just what defines frailty? J Am Geriatr Soc 2005;53:2229-2230.

18 Joseph B, Pandit V, Rhee P, Aziz H, Sadoun M, Wynne J, Tang A, Kulvatunyou N, O’Keeffe T, Fain MJ, Friese RS: Predicting hospital discharge disposition in geriatric trauma patients: is frailty the answer? J Trauma Acute Care Surg 2014;76:196-200.

19 Cuthbert JP, Corrigan JD, Harrison-Felix C, Coronado V, Dijkers MP, Heinemann AW, Whiteneck GG: Factors that predict acute hospitalization discharge disposition for adults with moderate to severe traumatic brain injury. Arch Phys Med Rehabil 2011;92: 721-730.e3.

20 Foreman BP, Caesar RR, Parks J, Madden C, Gentilello LM, Shafi S, Carlile MC, Harper CR, Diaz-Arrastia RR: Usefulness of the abbreviated injury score and the injury severity score in comparison to the Glasgow Coma Scale in predicting outcome after traumatic brain injury. J Trauma 2007;62:946-950.

21 Robinson TN, Wallace JI, Wu DS, Wiktor A, Pointer LF, Pfister SM, Sharp TJ, Buckley MJ, Moss M: Accumulated frailty characteristics predict postoperative discharge institutionalization in the geriatric patient. J Am Coll Surg 2011;213:37-42; discussion 42-44.

22 Lee DH, Buth KJ, Martin B-J, Yip AM, Hirsch GM: Frail patients are at increased risk for mortality and prolonged institutional care after cardiac surgery. Circulation 2010;121: 973-978.

23 Makary MA, Segev DL, Pronovost PJ, Syin D, Bandeen-Roche K, Patel P, Takenaga R, Devgan L, Holzmueller CG, Tian J, Fried LP: Frailty as a predictor of surgical outcomes in older patients. J Am Coll Surg 2010;210:901908.

24 Goldstein J, Andrew MK, Travers A: Frailty in older adults using pre-hospital care and the emergency department: a narrative review. Can Geriatr J 2012;15:16-22.

25 Hackbarth GM, Berenson R, Miller ME: Report to the congress: Medicare payment policy. Testimony on behalf of MedPAC, March $17,2009$.

26 Housley BC, Stawicki SP, Evans DC, Jones C: Comorbidity-polypharmacy score predicts readmission in older trauma patients. J Surg Res 2015;199:237-243.

27 Dent E, Hoogendijk EO: Psychosocial factors modify the association of frailty with adverse outcomes: a prospective study of hospitalised older people. BMC Geriatr 2014;14:108.
28 McAdams-DeMarco MA, Law A, Salter ML, Chow E, Grams M, Walston J, Segev DL: Frailty and early hospital readmission after kidney transplantation. Am J Transplant 2013;13:2091-2095.

29 Garonzik-Wang JM, Govindan P, Grinnan JW, Liu M, Ali HM, Chakraborty A, Jain V, Ros RL, James NT, Kucirka LM, Hall EC, Berger JC, et al: Frailty and delayed graft function in kidney transplant recipients. Arch Surg 2012;147:190-193.

30 Courtney-Brooks M, Tellawi AR, Scalici J Duska LR, Jazaeri AA, Modesitt SC, Cantrell LA: Frailty: an outcome predictor for elderly gynecologic oncology patients. Gynecol Oncol 2012;126:20-24.

31 Conroy S, Dowsing T: The ability of frailty to predict outcomes in older people attending an acute medical unit. Acute Med 2012;12: 74-76.

32 Hewitt J, Moug SJ, Middleton M, Chakrabarti M, Stechman MJ, McCarthy K; Older Persons Surgical Outcomes Collaboration: Prevalence of frailty and its association with mortality in general surgery. Am J Surg 2015;209: 254-259.

33 LaStayo PC, Ewy GA, Pierotti DD, Johns RK, Lindstedt S: The positive effects of negative work: increased muscle strength and decreased fall risk in a frail elderly population. J Gerontol A Biol Sci Med Sci 2003;58:M419_ M424.

34 Pijnappels M, van der Burg PJ, Reeves ND, van Dieën JH: Identification of elderly fallers by muscle strength measures. Eur J Appl Physiol 2008;102:585-592.

35 Moreland JD, Richardson JA, Goldsmith $\mathrm{CH}$, Clase CM: Muscle weakness and falls in older adults: a systematic review and meta-analysis. J Am Geriatr Soc 2004;52:1121-1129.

36 Wolfson L, Judge J, Whipple R, King M: Strength is a major factor in balance, gait, and the occurrence of falls. J Gerontol A Biol Sci Med Sci 1995;50(spec No):64-67.

37 Toosizadeh N, Mohler J, Wendel C, Najafi B: Influences of frailty syndrome on open-loop and closed-loop postural control strategy. Gerontology 2014;61:51-60.

38 Davis DH, Rockwood MR, Mitnitski AB, Rockwood K: Impairments in mobility and balance in relation to frailty. Arch Gerontol Geriatr 2011;53:79-83.

39 Delbaere K, Crombez G, Vanderstraeten G, Willems T, Cambier D: Fear-related avoidance of activities, falls and physical frailty. A prospective community-based cohort study. Age Ageing 2004;33:368-373.

40 Toosizadeh N, Najafi B, Reiman EM, Mager RM, Veldhuizen JK, O'Connor K, Zamrini E, Mohler J: Upper-extremity dual-task function: an innovative method to assess cognitive impairment in older adults. Front Aging Neurosci 2016;8:167.
Frailty Measurement to Predict Adverse Health Outcomes in Bedbound Elderly
Gerontology 2017;63:299-307 DOI: $10.1159 / 000453593$ 